\title{
Gendability - Gender und Diversity bewirken innovative Produkte
}

\author{
Dorothea Erharter, Elka Xharo
}

ZIMD Zentrum für Interaktion, Medien \& soziale Diversität

1

\section{Einleitung}

Frauen treffen einen Großteil der Kaufentscheidungen (Barletta, 2006; Klein-Franke, 2006, S. 80) und werden seit einiger Zeit auch als wichtige Zielgruppe für Unterhaltungselektronik und Kommunikationstechnologie betrachtet (Joost, 2008, S. 136).

Trotz des hohen Marktpotenzials scheint es, dass die tatsächlichen Wünsche und Bedürfnisse von Frauen selten vorab analysiert werden und in den Designprozess einfließen. Sehr viel häufiger werden Produkte für Frauen auf Basis von stereotypischen Zuschreibungen gestaltet.

Ähnliches lässt sich über ältere Menschen sagen. Sie übertreffen an Kaufkraft alle anderen Altersgruppen, wurden auch als Zielgruppe bereits prinzipiell entdeckt, und dennoch werden Produkte sehr selten an ihren Anforderungen und Bedürfnissen orientiert. Durch europaweite Förderschienen im Bereich Ambient Assisted Living (AAL) hat sich dies für den medizinnahen Bereich zwar mittlerweile gewandelt, in allen anderen Bereichen werden Senior_innen in die Produktentwicklung aber nur sehr selten einbezogen.

Es gibt also das Paradoxon, dass die Wirtschaft die Kaufkraft von Gruppen erkannt hat, sie als Zielgruppen erschließen möchte, dies aber nicht gelingt, weil diese Gruppen in der Produktentwicklung zu wenig berücksichtig werden.

Die Ursache scheint darin zu liegen, dass die technologischen Entwicklungen in Europa von relativ homogenen Teams aus Männern mittleren Alters dominiert werden, was dazu führt, dass vor allem die Bedürfnisse und Anforderungen dieser Gruppe berücksichtigt werden und andere Kund_innengruppen vernachlässigt werden (Joost, Bessing und Buchmüller, 2010, S. 17). Dies hat ernste Konsequenzen:

„It decreases the innovative power and inventiveness because of missing opponent, ambiguous or even conflicting viewpoints. It increases the pitfalls of 'I-methodologies' which means that the producers' assumptions become more or less consciously the leading benchmarks for technological developments instead of real users' needs and demands." (Buchmüller, Joost, Bessing und Stein, 2011, S. 744)

Harlfinger et al. haben gezeigt, dass (gut gemanagte) Diversität einer der wichtigsten Faktoren für Innovation ist (Rat für Forschung und Technologieentwicklung, FAS.research, 2008, S. 37). Joost et al. haben darauf hingewiesen, dass Frauen in mehrerer Hinsicht sehr anspruchsvolle Nutzerinnen von IKT sind, und daher neue oder andere Impulse für die allgemeine IKT-Entwicklung liefern können (Joost, Bessing und Buchmüller, 2010, S. 16, Buto- 
vitsch-Temm, 2008, S. 131 ff.). Die Einbindung von Frauen in den Innovationsprozess stellte sich also als ausgesprochen sinnvoll und fruchtbar heraus.

Genaue Kenntnisse der Kund_innen-Bedürfnisse sind einer der wichtigsten Erfolgsfaktoren für die Entwicklung neuer Produkte und Dienstleistungen (Joost, Bessing und Buchmüller 2010, S. 17).

Um das oben genannte Paradoxon aufzubrechen, muss also zum einen Diversity in die Design- und Entwicklungsteams Einkehr halten, und zwar in mehrerlei Hinsicht: Neben dem Geschlecht ist (u. a.) auch das Alter eine wichtige Innovationskategorie. In Thinking Aloud Tests entdecken Senior_innen mehr Gestaltungsfehler als alle anderen Gruppen. Die Bereinigung dieser Fehler ist aber in den meisten Fällen auch für alle anderen sinnvoll und bereichernd. Nur sehr wenige Fehler gehen auf echte „Alterserscheinungen“ zurück, also auf das Nachlassen des Muskeltonus, unbeweglichere/größere Finger oder darauf, dass unter den Seniorinnen und Senioren deutlich mehr Menschen nur eine vage Vorstellung von der Funktionsweise des Internets haben, Computer weniger nutzen und daher keine ausgeprägten mentalen Modelle von deren Abläufen haben.

Zum anderen müssen die zu erreichenden Zielgruppen nicht nur fiktiv (in Form bestehender Zuschreibungen), sondern real in die Produktentwicklungen einbezogen werden.

In diesem Artikel zeigen wir, wie durch die Berücksichtigung von Gender und Diversity die Qualität und die Usability von Produkten verbessert werden kann. Dafür hat Erharter (2012) den Begriff „Gendability“ geprägt. Wir arbeiten den Unterschied zwischen einem durch Stereotypen geleiteten Design und einem diversity-orientierten Ansatz heraus und zeigen anhand zweier Projekte, „G-U-T“ und „Mobi.senior.a“, was dies in der Praxis bedeuten kann.

\section{2 \\ Welche Aspekte sind für die Gestaltung von Websites und Apps von Bedeutung?}

Im Folgenden werden Aspekte dargestellt, die als Unterschiedskategorien zu beachten sind, um Usabilty und User Experience bei Websites und Apps angemessen zu gestalten. Es geht zunächst um die Diversity-Aspekte, die als mehr oder weniger veränderbare Dimensionen Unterschiedlichkeit herstellen. Dann werden Gender-Aspekte dargestellt und anschließend weitere Lebensrealitäten, die für die Human-Computer Interaction relevant sein können. Schließlich gehen wir auf Technikaffinität ein und unterscheiden hier zwischen quantitativen und qualitativen Nutzungsunterschieden. 


\section{$2.1 \quad$ Diversity-Aspekte}

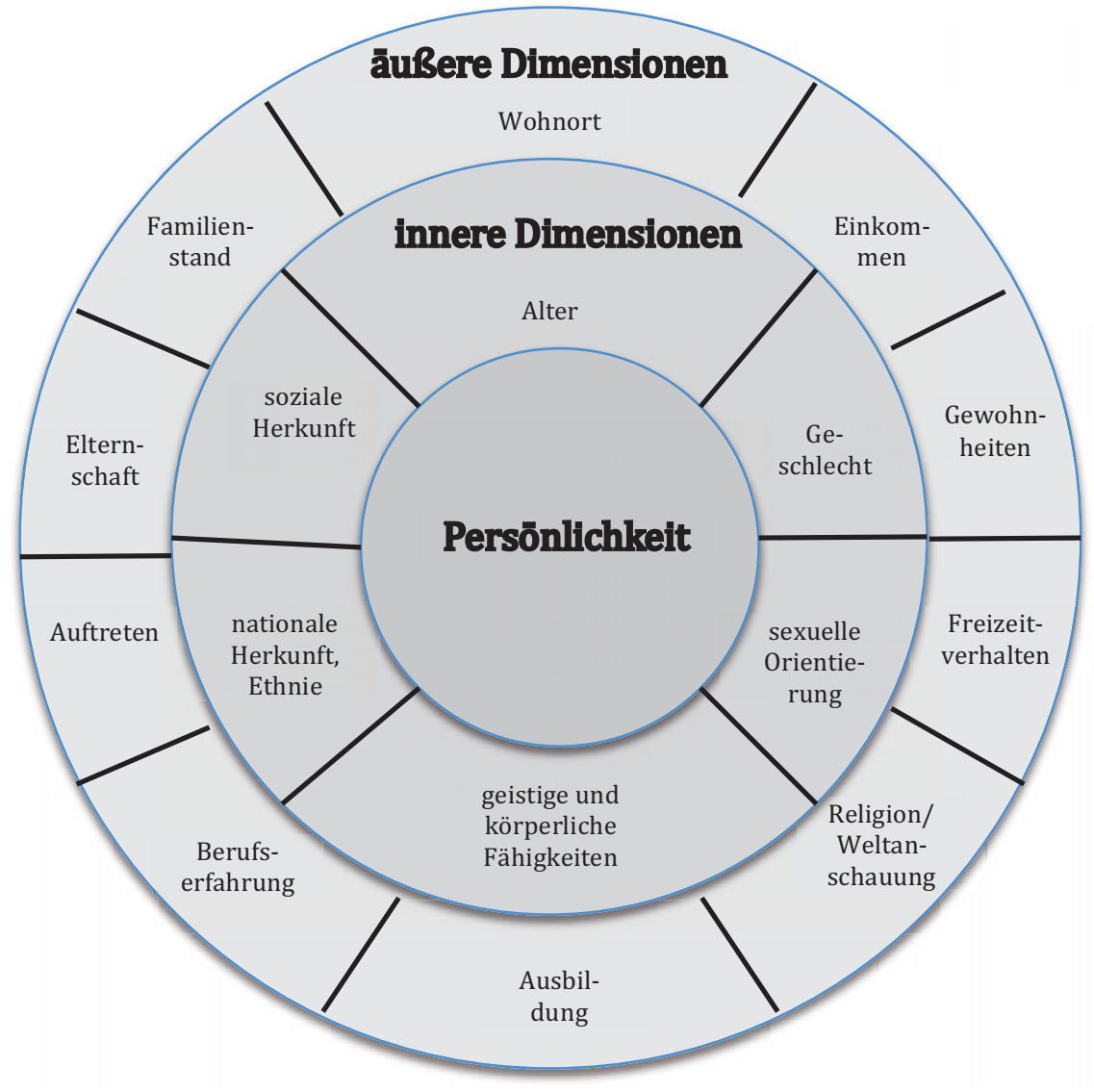

Abb. 1: Innere und äußere Dimensionen von Diversity in Anlehnung an Gardenswartz und Rowe (2003, S. 33)

Die Diversity-Dimensionen wurden (2003) von Gardenswartz und Rowe im obigen Diversity-Rad abgebildet. Es stellt die inneren (nicht oder kaum durch die Person veränderbaren) und die äußeren (leichter und häufiger veränderlichen) Dimensionen von Persönlichkeiten dar. Religion oder Weltanschauung ist sehr eng mit der Person verknüpft und daher zwar eine äußere, aber dennoch oft sehr eng mit der Persönlichkeit verknüpfte Dimension, dies ist durch die Farbgebung gekennzeichnet.

Die Diversity-Dimensionen eignen sich prinzipiell sehr gut für Zielgruppenüberlegungen zu Produkten im IKT-Bereich. Wichtig ist zu beachten, dass auf jeden Menschen immer alle Diversity-Merkmale gleichzeitig zutreffen und sich daher vielfältig überlagern. Im Design ständig alle Diversity-Dimensionen im Auge gleichzeitig zu behalten, wäre jedoch eine Überforderung.

Im Projekt „G-U-T“ haben wir eine Guideline für Entwickler_innen verfasst, wie Websites und Apps gender- und diversitygerecht gestaltet werden können. In diesem Zusammenhang haben wir durch vergleichende Analyse überprüft, welche Diversity-Dimensionen in der 
Praxis für das Design und Development von Apps und Websites relevant sind; bei welchen Design- und Entwicklungsschritten also durch welche Diversity-Dimensionen Veränderungen am Produkt zustande kommen können. Es hat sich gezeigt, dass nicht alle Dimensionen gleichermaßen relevant sind, sondern bestimmte Merkmale die Anforderungen an Apps und Websites erheblich stärker beeinflussen als andere.

Für das Design von Websites und Apps spielen demgemäß vor allem die folgenden Diversity-Dimensionen eine Rolle (Erharter, 2013, S. 9):

- Geschlecht

- Alter und Generationen

- $\quad$ Beruf bzw. Tätigkeitsfeld

- Technikerfahrung und -affinität (nicht im Diversity-Rad vorhanden)

- $\quad$ Bildung

- $\quad$ Ethnischer bzw. kultureller Hintergrund

- Beeinträchtigungen bzw. Behinderungen

Das Geschlecht spielt bei der Gestaltung von Websites vor allem bei der verbalen und der Bildersprache eine Rolle. Darüber hinausgehende Unterschiede kommen vor allem durch die unterschiedlichen Tätigkeitsfelder zustande, wie oben beschrieben.

Bei älteren Menschen nehmen physische Fähigkeiten schleichend ab. Manche sehen oder hören schlechter, können ihre Finger und Hände schlechter bewegen, haben eine schlechtere Konzentrationsfähigkeit etc., was die Computernutzung unmittelbar beeinflusst. Darüber hinaus haben sie häufig sehr wenig Erfahrung mit Computern und daher wenig Kompetenz im Umgang damit und weniger Hintergrundwissen. Vor allem dadurch tun sie sich in der Handhabung deutlich schwerer. Ältere Menschen haben aber auch manchmal andere Ansprüche an die Inhalte von Websites und Apps, zum Beispiel wünschen sie sich Erinnerungsfunktionen oder Gedächtnistraining. In Projekt „Mobi.senior.a“ führen wir dazu eine Studie durch, deren Ergebnisse aber noch nicht ausgewertet sind.

Das Tätigkeitsfeld beeinflusst die Lebensrealität der Menschen, das, was sie in ihrem Alltag und in ihrer Freizeit tun. Dazu gehören natürlich auch nicht-bezahlte Tätigkeiten, wie beispielsweise Kinderbetreuung oder ehrenamtliche Tätigkeiten. Daraus ergeben sich unterschiedliche Ansprüche und Erwartungen an Websites und Apps.

Die Technikerfahrung und -affinität ist eine von Erharter dem Diversity-Rad hinzugefügte „äußere“ Kategorie, die sich im Laufe des Lebens ändern kann. Sie bezieht sich auf die grundlegende Bereitschaft und Motivation, sich mit technischen Dingen zu befassen und auf technische Vorerfahrungen und Kompetenz.

Bildung und ethnischer Hintergrund wirken sich vor allem auf das Sprachenverständnis aus und teilweise auch darauf, wie Abbildungen von Kulturgütern wahrgenommen, und was als exklusiv bzw. inklusiv erlebt wird.

Beeinträchtigungen und Behinderungen beeinflussen vor allem die Art und Weise, wie etwas genutzt wird. Bereits eine leichte Sehschwäche kann beispielsweise bewirken, dass Smartphones unterwegs weniger genutzt werden, da das Hervorkramen der Brille als umständlich erlebt wird.

Allen Dimensionen gemeinsam ist, dass sie sich durch die unterschiedlichen Lebensrealitäten der Personen auf die Nutzungsszenarien und Abläufe auswirken.

Näheres dazu auch unter http://g-u-t.zimd.at. 


\section{$2.2 \quad$ Gender-Aspekte}

In der Entwicklung von Websites und Apps sind Gender-Aspekte im engeren Sinne vor allem für eine integrierende und diskriminierungsfreie Gestaltung von Bedeutung. Dies betrifft zum einen geschlechtssensible Sprache und zum anderen die Bildsprache.

In der Auswahl der Bilder sollen Stereotypen vermieden werden und Menschen gleichwertig und realistisch in ihrem Lebensalltag dargestellt werden, anstatt, wie es häufig der Fall ist, Menschen übertrieben (auch überhöht) darzustellen, denn letzteres führt zu einer Reduktion der Menschen auf eine Eigenschaft oder Rolle, und wird den realen Menschen nicht gerecht. Sie können sich damit nicht identifizieren.

Das bedeutet nicht, dass gleichgemacht werden soll, sondern kann zu einem spielerischen Umgang mit Alltagssituationen führen. Meist werden die Darstellungen dadurch interessanter, weil Menschen in ihrem Tun mehrdimensional dargestellt werden. Dies gilt nicht nur für das Geschlecht, sondern auch für die Dimensionen Alter, Behinderungen, kultureller oder religiöser Hintergrund und sexuelle Orientierung.

Wie dies ausssehen kann, zeigen zum Beispiel die ider Wiener Linien, dem Betreiber des öffentlichen Nahverkehrs der Stadt Wien. In dieser Werbung schmunzeln beispielsweise zwei ältere Frauen kopfschüttelnd über „Diese Jugend von heute“, als ein junger Mann ihnen einen Platz anbietet. Oder es steigen zwei Männer in eine Bahn ein, in der eine attraktive Frau steht, und der eine ermahnt den anderen „Jetzt heißt's Bauch einziehen“.

\section{$2.3 \quad$ Lebensrealitäten}

Darüber hinausgehende Unterschiede, die sich auf das Design von Websites und Apps auswirken können, kommen weniger durch das Geschlecht als durch unterschiedliche Lebensrealitäten zustande.

„Signifikante Unterschiede kommen erst durch die unterschiedlichen Lebenssituationen von Frauen und Männern zustande. Beispielsweise ist das Merkmal ,Frau' erheblich häufiger mit dem Merkmal ,Betreuungspflicht für Kinder unter 14 Jahre` verknüpft. Geschlecht allein macht noch keinen Unterschied, es sind die Kinder, die ihre Wirkung auf die alltäglichen Wege entfalten und signifikant auf Geschlechterunterschiede wirken.“ (Erharter, 2012, S. 6; vgl. Scambor und Zimmer, 2012)

Frauen haben häufig vielfältigere Lebensbereiche, mehr und unterschiedlichere Alltagsdestinationen und haben weniger Freizeit, sie stehen also mehr unter Zeitdruck (Scambor und Zimmer, 2012).

In der Design-Praxis bedeutet dies: Nutzungsszenarien und nützliche Funktionalitäten sind nicht $\mathrm{zu}$ vermuten, sondern zu erfragen bzw. zu beobachten. Sie können nicht durch „I-Methodology“ (Bath 2009, S. 125 ff.), sondern nur durch Einbindung realer Nutzer_innen in den Design-Prozess herausgefunden werden.

\subsection{Technikaffinität}

Darüber hinaus gibt es quantitative Nutzungsunterschiede nach Geschlecht und Alter, die die mittlere Technikaffinität der Gruppen und ihre Erfahrung mit technischen Medien abbilden. 


\section{Quantitative Nutzungsunterschiede}

Die absoluten Zahlen haben sich seit 2008 verändert, doch nach wie vor gibt es in der Internetnutzung relativ große Unterschiede zwischen Frauen und Männern (Deutschland 2013: $9,6 \%$, Initiative 21,2013 ) und sehr große Unterschiede zwischen den Altersgruppen (Deutschland 2013: ca. $67 \%$, ebenda).

Die folgende Grafik auf Basis der Zahlen vom (N)Onliner-Atlas 2008 (Initiative 21, 2008, S. 12) zeigt die Nutzungsunterschiede zwischen Männern und Frauen verschiedener Altersgruppen und Migrationserfahrungen. Die größten Unterschiede zwischen Männern und Frauen finden sich in der Altersgruppe 50 Plus, und dort gibt es auch relativ große Unterschiede zwischen Gruppen ohne/mit eigener/mit Migrationserfahrung der Eltern. In der mittleren Altersgruppe fällt der ebenfalls niedrigere Online-Anteil von Frauen gegenüber Männern auf, und auch hier relativ große Unterschiede nach Migrationserfahrung.

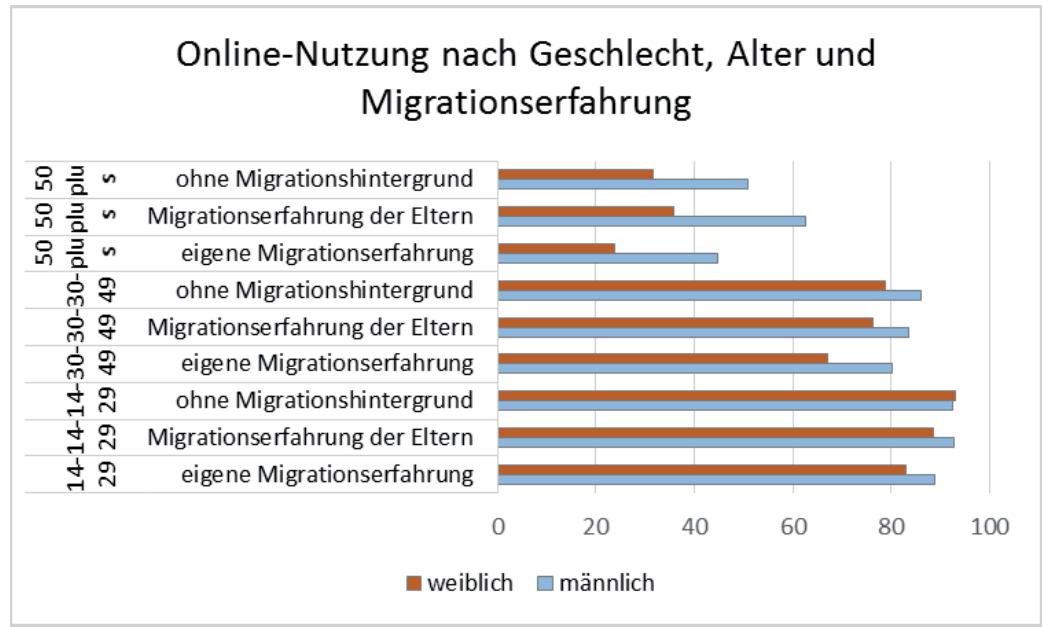

Abb. 3: Nutzungsunterschiede zwischen Frauen und Männern verschiedener Altersgruppen und Migrationserfahrungen. Eigene Grafik auf Basis der Zahlen vom (N)Onliner-Atlas 2008 (Initiative 21, 2008, S. 12)

Lediglich in der Gruppe der 14-29-Jährigen ohne Migrationserfahrung haben die jungen Frauen die Männer überholt. In den beiden Gruppen mit eigener Migrationserfahrung bzw. mit Migrationserfahrung der Eltern sind die Unterschiede gegenüber den anderen Altersgruppen geringer.

In der Gruppe der Senior_innen existieren große genderspezifische Unterschiede in den Anforderungen digitale Medien und Motivationen, diese zu nutzen. Ältere Frauen sind häufig sowohl mit den Ausschlusseffekten des Doing Gender als auch des Doing Aging konfrontiert (Haring, 2011, S. 2). Unter den Senior_innen haben mehr Männer internetfähige Mobiltelefone und nutzen sie häufiger - der Unterschied wird aber geringer (Mohr, Sauthoff-Bloch, Alt und Derksen, 2011, S. 11).

\section{Qualitative Nutzungsunterschiede}

Es ist zu beachten, dass die Zielgruppe der Senior_innen nicht homogen ist. Es gibt Bereiche, die von Frauen öfter genutzt werden als von Männern. „Frauen nutzen häufiger als Männer 
mobile Social-Web-Anwendungen und bewegen sich häufiger in Online Communities. " (Mohr, Sauthoff-Bloch, Alt und Derksen, 2011, S. 11).

Auch unter Jugendlichen gibt es qualitative Unterschiede in der Nutzung. Mädchen verwenden das Internet zum Beispiel vergleichsweise häufiger als Kommunikationsmedium und sind damit auch regelmäßiger und länger in sozialen Netzwerken unterwegs (BITKOM, 2011, S. 1).

Diese Unterschiede im kommunikativen Onlineverhalten wurden von unserer eigenen Studie im Projekt „Mobi.senior.a“ für Senior_innen bestätigt. Knapp die Hälfte der älteren Frauen, aber kein einziger Mann gaben an, dass ihnen auf mobilen Geräten die Nutzung sozialer Netzwerke eher oder sehr wichtig sei, Ähnliches gilt für „WhatsApp“ (7 von 15 Frauen, 1 Mann von 12 Männern) (Erharter und Xharo, 2014).

In „Mobi.senior.a“ fragten wir 15 Frauen und 12 Männer, welche Funktionen auf Mobiltelefonen ihnen wie wichtig wären. Dies ist in quantitativer Hinsicht keine repräsentative Stichprobe, es ging hier darum, die qualitativen Aspekte heraus zu arbeiten. Die möglichen Funktionen wurden aufgrund einer Vorbefragung unter 12 Nutzer_innen aller Altersgruppen von uns vorgegeben und konnten durch die Testpersonen ergänzt werden. Die Funktionen wurden dabei verschiedenen Kategorien zugeordnet: Kommunikation | Etwas nachschauen, das ich gerade brauche, mit aktuellem Ort | Etwas nachschauen, das ich gerade brauche, ohne aktuellem Ort | Zeitvertreib | Fotos | Etwas kontrollieren | Etwas bearbeiten. Die Testpersonen konnten in jeder Kategorie eigene Ergänzungen hinzufügen. Die einzige Ergänzung, die von mehr als zwei Testpersonen genannt wurde, war „WhatsApp“.

Die folgenden beiden Diagramme zeigen eine Auswahl der Funktionen, und zwar in der oberen Grafik solche, die von mehr Frauen für eher oder sehr wichtig befunden wurden, und in der unteren Grafik von mehr Männern. Aus Gründen der Vergleichbarkeit wurden die absoluten Zahlen (15 Frauen, 12 Männer) in Prozentzahlen umgerechnet.

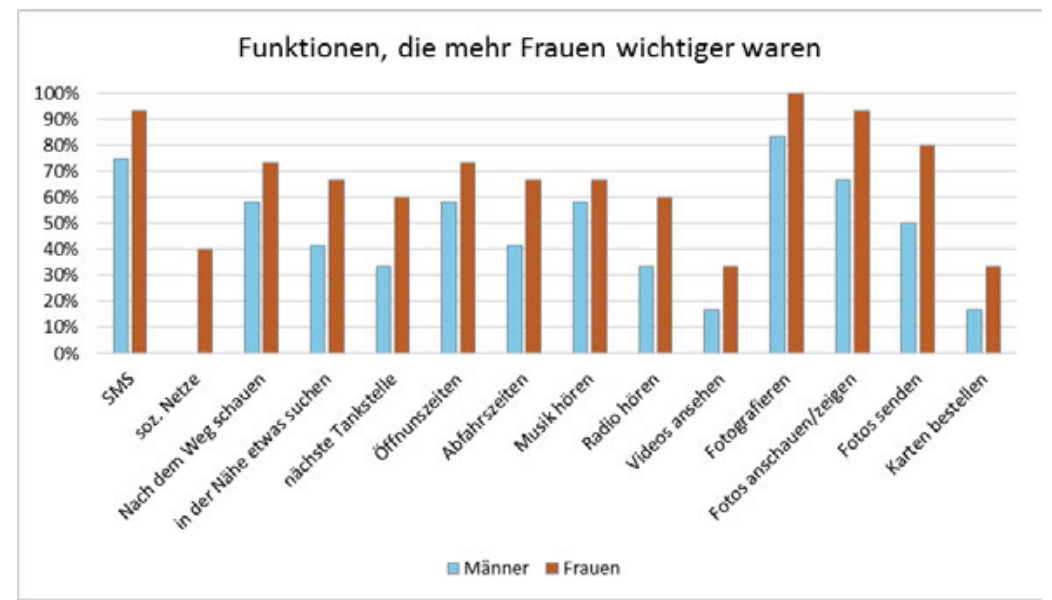

Abb. 4: Funktionen, die mehr Frauen wichtig waren als Männern. Eigene Grafik, Projekt „Mobi.senior.a“. (N=12 Männer, 15 Frauen) 


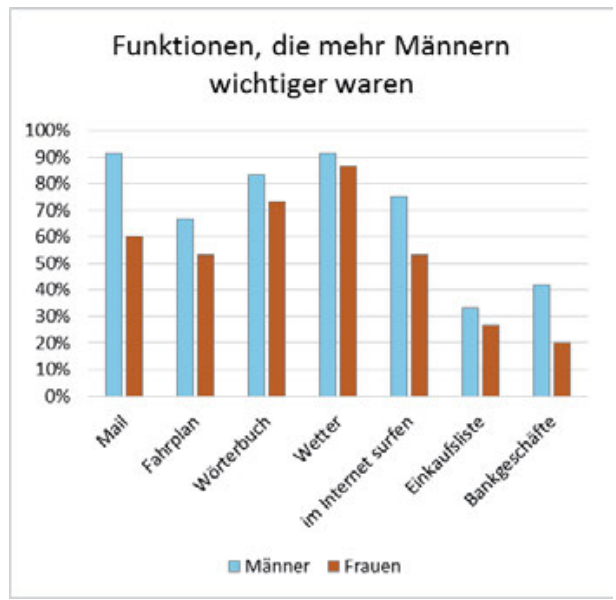

Abb. 5: $\quad$ Funktionen, die mehr Männern wichtig waren als Frauen. Eigene Grafik, Projekt „Mobi.senior.a“. (N=12 Männer, 15 Frauen)

\section{Der Design-Prozess}

Im Rahmen des Prozesses der Gestaltung gilt es, mit der eigenen Rolle und der Zielgruppe reflektiert umzugehen und die Nutzerinnen und Nutzer am Gestaltungsprozess teilhaben zu lassen. Im folgenden werden die Punkte dargestellt, die für eine Integration von Diversity in den Design-Prozess relevant sind. Dabei geht es zunächst um Vorurteile und Selbstreflexion, dann um Zielgruppen-Reflexion und schließlich um partizipatives Design.

\subsection{Vorurteile und Selbstreflexion}

Vorurteile sind unreflektierte Annahmen über andere Personen. Sie haben eine wichtige lebenspraktische Funktion. Wenn unmittelbare Gefahr droht, muss diese reflexhaft rasch erkannt werden, um blitzschnell reagieren zu können. Dies ist die Funktion von vorgefassten Meinungen, sogenannten Vorurteilen.

Wenn aber keine unmittelbare Gefahr droht, und das trifft in einer zivilisierten Gesellschaft wie der unseren meist zu, sind Vorurteile ein Hindernis, denn sie verhindern einen differenzierten Blick auf das Gegenüber, auf seine/ihre Vorzüge, Schwächen, Anforderungen und Bedürfnisse.

Wir verwenden bewusst das Wort „Vorurteile“ und nicht „Vorannahmen“ oder Ähnliches, um dieses - in der Alltagssprache geläufigere - Wort zu enttabuisieren, und auch um verständlich zu machen, welche Reflexion wir befürworten. Denn um Vorurteile zu reflektieren, ist es wichtig, sie sich einzugestehen und bewusst zu machen. Denn wenn es darum geht, differenzierte Entscheidungen zu treffen, sind Vorurteile im Weg.

Die Selbstreflexion über die eigenen Vorurteile und Stereotypien ist die Basis jeglicher Zielgruppen-Reflexion. In der Design-Praxis droht keine unmittelbare Gefahr. Dennoch ist der 
Design-Prozess häufig eher von Stereotypen geprägt als von echtem Wissen über die Zielgruppe. Joost hat beschrieben, dass die Gruppe von Nutzerinnen bzw. Nutzern, die ein Produkt erreichen soll, sehr genau eingegrenzt und untersucht werden muss, um Pauschalierungen zu entgehen und ihre Bedürfnisse, Abneigungen, Fähigkeiten und Kontexte zu verstehen (Joost, 2008). Auch im Projekt „G-U-T“ hat sich gezeigt, wie wichtig eine genaue und strukturierte Zielgruppen-Reflexion ist:

Die Zielgruppen können damit eingegrenzt und genauer beschrieben, aber auch Zielgruppen neu entdeckt werden, an die man sonst nicht gedacht hätte. Das Diversity-Rad bzw. obige Dimensionen können dabei hilfreich sein. Sehr wichtig ist dabei die Reflexion der eigenen Zuschreibungen und Stereotypen (Bührer und Schraudner, 2006).

Einen großen Einfluss auf die Nutzung von Geräten und Software aller Art haben physiologische Unterschiede. Im Projekt „Mobi.senior.a“ haben wir die für die Nutzung von Smartphones/Tablets bedeutendsten Beeinträchtigungen identifiziert:

- Sehschwäche

- Farbensehen

- Hörbeeinträchtigungen

- Hände: Zittern

- Hände: Unbeweglichkeit/Schmerzen

- Hände: dickere Finger

- Hände: nachlassender Muskeltonus

In welcher Form diese Punkte für die Design-Praxis relevant sind, wird im unten aufgeführte Beispiel näher dargestellt.

\subsection{Partizipatives Design}

Mit partizipativen Methoden können Bedürfnisse aus der alltäglichen Realität der Kund_innen heraus erfasst werden. Neben text-orientierten Methoden wie Interview oder Fokusgruppen, mit denen bewusste Informationen gut erfasst werden können, eignen sich assoziativere bzw. kreativere Methoden wie Rollenspiele oder Cultural Probes, um tiefere Wissensschichten freizulegen. Sie eignen sich besonders zur Kreation neuer Ideen und Anwendungsmöglichkeiten. (Joost, Bessing und Buchmüller, 2010, S. 20)

Die klassischen Usability-Methoden, wie Thinking Aloud Tests, Card Sorting oder Personas liegen dazwischen. Mit ihnen wird das Tun erfasst und damit unbewusste Hürden und teilbewusste Bedürfnisse und Anforderungen an vorhandene Anwendungen oder Prototypen sichtbar gemacht.

„Insgesamt ist von Bedeutung, nicht nur von Männern und Frauen auszugehen, sondern von Menschen mit vielen verschiedenen Merkmalen und in vielen verschiedenen Nutzungskontexten, da sonst erst recht wieder die Gefahr der Stereotypisierung besteht. Davon profitieren auch Männer, wie Raewyn Connell gezeigt hat (2006), die von verschiedenen ,Männlichkeiten“ spricht.“(Erharter, 2012. S. 113)

Ein sehr gutes Beispiel, wie dies in der Praxis umgesetzt werden kann, hat Andrea Wolffram (Wolffram, 2006, S. 19 ff.) gezeigt, die in einer Studie Technikhaltungen von Studienanfänger_innen untersucht hat: Sie hat nicht von vorneherein zwischen Frauen und Männern unterschieden und die Technikhaltungen dieser beiden (vorgegebenen) Gruppen untersucht, 
sondern ist den umgekehrten Weg gegangen: Sie hat zuerst die Technikhaltungen geclustert, und erst danach (!) die Geschlechterverteilungen innerhalb der fünf Gruppen betrachtet. Mit einer solchen Vorgangsweise fällt es deutlich leichter, der Falle vorschneller Zuschreibungen zu entgehen.

Im partizipativen Design bedeutet dies: Die Testpersonen müssen anhand von Gender- und Diversity-Merkmalen ausgewählt werden. Bei den Aufgabenstellungen sind insbesondere die unterschiedlichen Lebensrealitäten und Nutzungsszenarien zu berücksichtigen. Vergleichende Fragestellungen oder Auswertungen zwischen vorgegebenen Gruppen („Frauen und Männer", Altersgruppen) sind aber möglichst zu vermeiden bzw. selbstkritisch zu betrachten. Günstiger ist es, auf Basis der Fragestellungen neue Gruppen zu bilden (,Menschen mit Betreuungspflichten“), die dann Frauen und Männer bzw. Menschen verschiedener Altersgruppen umfassen.

Mit der G-U-T-Checklist hat Erharter (2013) Materialien entwickelt, die im Design-Prozess genutzt werden können, um durch Gender, Diversity und Usability die Qualität von Websites und Apps zu verbessern. Es ging bei dem Projekt neben der Zusammenführung und Strukturierung großteils bereits bekannter Inhalte vor allem um den Transfer zur Zielgruppe der Entwickler_innen und Designer_innen, also darum, die Inhalte für sie verständlich zu vermitteln.

Die G-U-T-Checklist umfasst:

- eine Guideline, in der die Inhalte erläutert werden,

- eine Anleitung zur Selbstreflexion,

- $\quad$ eine Checklist für Apps und Tablets und

- $\quad$ eine Checklist für Websites.

$\mathrm{Zu}$ finden ist die G-U-T-Checklist unter http://g-u-t.zimd.at/content/gut-checklist.

\section{$5 \quad$ Mobi.senior.a: Gender-Aspekte in einem Projekt}

„Innovation ist die Kunst, sich die Dinge wieder aus dem Kopf zu schlagen.“

Wolfgang Pekny

In diesem Abschnitt zeigen wir, was Gendability in einem Projekt konkret bedeuten kann. Im Projekt „Mobi.senior.a“ erforschen wir insbesondere, welche Bedürfnisse und Anforderungen Seniorinnen und Senioren an Smartphones und Tablets und insbesondere an Apps haben. Bislang wurden 26 Thinking Aloud Tests mit dieser Zielgruppe durchgeführt, deren Ergebnisse hier einfließen.

Gender- und Diversity-Aspekte sind bereits im Forschungsdesign berücksichtigt, indem beispielsweise der Fokus nicht nur auf Usability-Hürden, sondern auch auf Technikhaltungen im Sinne des Doing Gender und Doing Aging gelegt wird. Auch in der Literaturrecherche werden Zusammenhänge dieser beiden Aspekte und Überschneidungen mit anderen Merkmalen berücksichtigt. 
Im konkreten Studiendesign fließen Gender- und Diversity-Aspekte in der Auswahl und Zusammensetzung der Proband_innen nicht nur nach Geschlecht und Alter, sondern auch nach Technikdistanz und Milieu ein. Es wird versucht, möglichst unterschiedliche Lebenskontexte zu berücksichtigen. Erforscht wird durch das ZIMD

- die Usability von Apps,

- Usability-Hürden bei der ersten Inbetriebnahme von Smartphones und Tablets,

- welche Funktionen am Smartphone/Tablet für ältere Menschen sinnvoll sein können (Usefulness).

Bei den ersten beiden Fragestellungen geht es vor allem um Hürden bei der Benutzung, und die sind - von Design-Fehlern einmal abgesehen - großteils von Vorerfahrungen, dem grundlegenden Verständnis von Internet und Smartphones und der Experimentierfreudigkeit der Nutzer_innen abhängig. Hier spielt bei einigen Senior_innen auch das Doing Aging eine gewisse Rolle, die aber nicht überschätzt werden sollte. Die von uns befragten Senior_innen waren teilweise ausgesprochen experimentierfreudig.

Insbesondere mit der letzten Forschungsfrage versuchen wir, zum einen die Lebensrealitäten der (sehr unterschiedlichen) Seniorinnen und Senioren abzubilden, und darüber hinaus sie intensiv zur Ideenfindung für ihren eigenen Alltag anzustiften. Dies erfolgt in Form einer Tagebuch-Studie, die wir für diesen Zweck abgewandelt und erweitert haben.

\section{Ergebnisse des Projekts}

Die meisten Usability-Probleme, die wir gefunden haben, treffen nicht nur auf Senior_innen zu, sondern können sich ohne weiteres verallgemeinern lassen. Es zeichnet sich jedoch ab, dass vorhandene Usability-Probleme für Senior_innen - aufgrund eines geringeren Erfahrungsschatzes im Umgang mit Smartphones/Tablets und mit Computern insgesamt - sich für viele Senior_innen störender auswirken als für andere, sie also an solchen Hürden eher scheitern. Nur wenige Usability-Probleme gehen auf die oben beschriebenen Alterserscheinungen zurück. Häufiger ist eine Kombination: Vorhandene Usability-Probleme, wie zu kleine Buttons, sind für Senior_innen mit leichten motorischen Problemen oder eine leichten bis mittleren Fehlsichtigkeit eine größere Hürde als für andere. Die vorgeschlagenen Gestaltungsrichtlinien für die Entwicklung von Apps aus Gender- und Diversity-Sicht sind also für alle Zielgruppen sinnvoll und für die Zielgruppe(n) der Senior_innen von besonders großer Bedeutung.

Im Folgenden fassen wir die ersten Ergebnisse aus den Usability-Studien (Mobi.senior.a) zusammen. Ausführliche Beschreibungen finden sich im Tagungsband zum Usability-Day XII der Fachhochschule Dornbirn, im Artikel „Smartphones, Tablets, App für Seniorinnen und Senioren" (Erharter und Xharo, 2014).

Prinzipiell gilt: Es sollen auch auf Websites nur Funktionen angeboten werden, die für die Anwender_innen wirklich einen Nutzen bringen. Dies gilt für Apps und mobile Websites in noch radikalerer Weise.

\section{Größe und Kontrast}

Es sollte selbstverständlich sein, ist es aber nicht: Buttons und Beschriftungen müssen für mobile Nutzung größer sein und größere Abstände haben, zum einen, da die Nutzung häufig in störanfälligerer Umgebung und mit weniger Aufmerksamkeit erfolgt, zum anderen, wenn 
ältere Menschen zur Zielgruppe zählen, da diese häufiger motorische Schwierigkeiten haben.

Ein guter Kontrast ist erforderlich, damit Elemente erkannt werden.

\section{Interaktionsdesign}

- Modale Dialoge, die den Hintergrund inaktiv setzen, führen meist zu viel Verwirrung bei Senior_innen, da nicht verstanden wird, warum die Felder des Hintergrunds nicht mehr funktionieren.

- Der sich schnell ausschaltende Bildschirm war für viele Senior_innen ein Problem, da sie eher langsam mit dem Gerät agiert haben.

- Der Bildschirmschoner sorgte bei manchen Senior_innen für Verwirrung.

- Das Fehlen von Bestätigungs-Buttons führte zu Verwirrung. Wichtig sind klare, gut erkennbare OK-Buttons, rechts unter dem, was zu bestätigen ist.

- Inkonsistentes Design ist immer zu vermeiden: Wiederkehrende Navigationselemente sollen immer an derselben Stelle sein und dasselbe Design haben.

\section{Navigationselemente}

- Vor allem nach einer unbeabsichtigten Aktion wünschen sich die Testpersonen eine Zurück-Taste, um den Fehler rückgängig zu machen.

- Es gibt so etwas wie „Bannerblindheit“: Schaltflächen im oberen Bereich des Bildschirms unter einem Bild, aber auch Menüleisten (mit schwachen Kontrasten) im unteren Bereich des Bildschirms wurden schlecht erkannt.

- Buttons („Filter“ und ,,+“) wurden eher gefunden, wenn sie umrandet und hervorgehoben und nicht nur flach als Schriftzug dargestellt waren.

- Die meisten Testpersonen navigieren eher mit Auswahllisten als mit Wischgesten.

- Navigationselemente, die es anzutippen gilt, sollten möglichst groß gestaltet sein.

- Das System von Checkboxen führt bei vielen Senior_innen zu Verwirrung, vor allem, wenn die Checkboxen bereits angehakt sind. Die meisten wollen die gewünschte Kategorie antippen, um sie anzuzeigen.

\section{Steuerung: Gesten}

- Wischgesten nach unten werden nur gemacht, wenn es Anzeichen dafür gibt, dass die Seite länger ist.

- Die Wischgesten zum Verkleinern/Vergrößern werden von vielen Senior_innen gekannt und intuitiv genutzt.

- Viele Senior_innen tippen entweder zu lang oder mit dem Fingernagel. Auch ein nicht notwendiger Druck wird mit dem Finger ausgeübt.

\section{Bildsprache}

Bilder ziehen die Aufmerksamkeit auf sich und können Informationen, aber auch Haltungen und Werte in kürzester Zeit transportieren. Eine besondere Bedeutung liegt aus Gender- und Diversity-Sicht in der Darstellung von Menschen, da Bilder die Wirklichkeit nicht nur abbilden, sondern auch das Bild der Wirklichkeit prägen, das Menschen haben. Bilder sind immer auch Deutungen.

In der Bildauswahl soll daher bewusst darauf geachtet werden, Stereotypen zu vermeiden, insbesondere hinsichtlich Geschlecht, Alter, Behinderungen, kulturellem oder religiösem 
Hintergrund und sexueller Orientierung. Das bedeutet nicht, dass gleichgemacht werden soll, sondern kann zu einem spielerischen Umgang mit Alltagssituationen führen. Meist werden die Darstellungen dadurch interessanter, weil Menschen in ihrem Tun mehrdimensional dargestellt werden. Ein guter Leitfaden zu diesem Thema ist www.vielefacetten.at (Knoll, 2014).

\section{Sprache}

- $\quad$ Englische Begriffe, wie „Return“, werden oft nicht verstanden.

- Fachbegriffe und die meisten Erklärungen beim Einrichten eines Gerätes werden nicht verstanden.

\section{Labels}

Bezeichnungen von Navigationselementen müssen der Erwartungshaltung entsprechen. Eine gute Möglichkeit, die Erwartungen abzuklären, sind Card Sorting Tests.

\section{Icons}

- Icons werden häufig nicht erkannt oder nicht verstanden.

- Von relativ vielen intuitiv verstanden wurde das Mehr-Personen-Icon für Mail/soziale Netze.

- Bereits bekannte Icons werden oft eher aufgrund ihrer Positionierung als aufgrund ihres Aussehens angeklickt. Es ist daher absolut notwendig, in der Anordnung und Lage der Icons konsistent zu bleiben.

\section{Nutzer_innen-Eingaben}

- Viele Senior_innen wissen nicht, dass man zur Eingabe in ein Textfeld hinein tippen muss, damit die Tastatur erscheint.

- Fast alle Senior_innen hatten ein Problem mit den zu kleinen Tasten zur Texteingabe.

- Viele Testpersonen haben Probleme mit der Navigation des Cursors und wünschen sich Pfeiltasten wie beim PC.

- Die Eingabefelder sollten möglichst fehlertolerant sein und Suchergebnisse während der Eingabe anzeigen.

- Bei Eingabemöglichkeit im oberen Bereich des Bildschirms wurde die im unteren Bereich befindliche Tastatur von einigen Testpersonen nicht in Zusammenhang mit der Eingabemöglichkeit gebracht.

\section{$6 \quad$ Fazit}

Gendability bedeutet also die gender- und diversitygerechte Gestaltung von IKT-Produkten (hier am Beispiel vom Apps und Websites), mit besonderem Augenmerk auf die Vermeidung von Hürden, also der Usability. Quantitative und qualitative Nutzungsunterschiede entstehen durch unterschiedliche Technikhaltungen und sind geprägt durch Doing Gender und vor allem durch Doing Aging.

Für Gendability ist zunächst die Reflexion der eigenen Vorannahmen (,Vorurteile“) von Bedeutung, um frei zu werden von stereotypen Zuschreibungen. Auf dieser Basis ist eine 
genaue Analyse der Zielgruppen sinnvoll, mit der oft auch neue Zielgruppen erschließbar werden. Wir schlagen vor, die Zielgruppen anhand der Diversity-Merkmale zu analysieren. Um die realen Anforderungen und Bedürfnisse unterschiedlicher Zielgruppen zu erfassen und einfließen lassen zu können, ist die Einbindung von Nutzer_innen im Sinne des partizipativen Designs wesentlich.

Beim Design von Produkten zu berücksichtigen sind insbesondere:

- die Diversity-Dimensionen Geschlecht, Alter und Generationen, Beruf bzw. Tätigkeitsfeld, Technikerfahrung und -affinität (nicht im Diversity-Rad vorhanden), Bildung, Ethnischer bzw. kultureller Hintergrund, Beeinträchtigungen bzw. Behinderungen,

- $\quad$ eine integrierende und diskriminierungsfreie verbale und Bildsprache,

- die Berücksichtigung der realen Nutzungskontexte und des Lebensalltags der Nutzer_innen.

Dadurch können neue Zielgruppen erschlossen, Produktideen generiert und ausgestaltet, deren Treffsicherheit überprüft und nicht zuletzt eine mehrdimensionale Bildsprache entwickelt werden. Für uns sind Geschlecht und Alter damit zu kaum zu überschätzenden Innovationskategorien geworden.

\section{Literatur}

Barletta, M. (2006) Marketing to Women; Berkshire: Kaplan Publishing.

Bath, C. (2009). De-Gendering informatischer Artefakte. Grundlagen einer kritisch-feministischen Technikgestaltung (Dissertation). Bremen: Staats- und Universitätsbibliothek Bremen. URN: http://nbn-resolving.de/urn:nbn:de:gbv:46-00102741-12

BITKOM (Hrsg.) (2011). Jugend 2.0 - Eine repräsentative Untersuchung zum Internetverhalten von 10- bis 18-Jährigen. Abgerufen am 28. Februar 2014 von http://www.bitkom.org/files/documents/BITKOM_Studie_Jugend 2.0.pdf

Buchmüller, Sandra, Joost, Gesche, Bessing, Nina \& Stein, Stephanie (2011). Bridging the gender and generation gap by ICT applying a participatory design process. Personal and Ubiquitous Computing (15)7, 743-758.

Bührer, Susanne \& Martina Schraudner (2006). Wie können Gender-Aspekte in Forschungsvorhaben erkannt und bewertet werden? Stuttgart: Fraunhofer IRB Verlag.

Butovitsch-Temm, Tatiana (2008). If You Meet the Expectations of Women, You exceed the Expectations of Men. How Volvo Designed a Car for Women Customers and Made World Headlines. In L. Schiebinger (Hrsg.), Gendered Innovations in Science and Engineering (S. 131-149). Stanford: Stanford University Press.

Connell, Raewyn (2006). Der gemachte Mann: Konstruktion und Krise von Männlichkeiten. Frankfurt a. M.: VS Verlag für Sozialwissenschaften.

Erharter, Dorothea, Xharo, Elka (2014). Smartphones, Tablets, App für Seniorinnen und Senioren. In Guido Kemper (Hrsg.), Tagungsband zum uDay XII am 16. Mai 2014 an der Fachhochschule Vorarlbert, Österreich.

Erharter, Dorothea (2013) G-U-T Guideline. Abgerufen am 28. Februar 2014 von http://g-ut.zimd.at/sites/default/files/files/GUT-A-Guideline.pdf

Erharter, Dorothea. (2012). Gendability - Was hat Usability mit Gender zu tun? In G. Kempter, K.H.Weidmann (Hrsg.), Technik für Menschen im nächsten Jahrzehnt. Beiträge zum Usability Day X (S 107-117). Lengerich: Pabst Science Publ. 
Gardenswartz, Lee \& Rowe, Anita (2003). Diverse Teams at Work. Alexandria, Virginia: Society for Human Resource Management.

Haring, Solveig (2011). Neue Medien ,alte“ Frauen. Medienkompetenz für ein Aufweichen von Klischees. In Magazin erwachsenenbildung.at. Das Fachmedium für Forschung, Praxis, und Diskurs, 13. Abgerufen am 21. Dezember 2012 von http://www.erwachsenenbildung.at/magazin/11-13/meb1113.pdf

Initiative D21 (Hrsg.) (2008). (N)Onliner Atlas. Sonderauswertung Internetnutzung und Migrationshintergrund in Deutschland. Abgerufen am 28. Januar 2014 von http://www.initiatived21.de/wpcontent/uploads/alt/08_NOA/NOA_Migration.pdf

Initiative D21 (Hrsg.) (2013). (N)Onliner Atlas. Abgerufen am 14. Mai 2014 von http://www.initiatived21.de/portfolio/nonliner-atlas/

Joost, Gesche, Bessing, Nina \& Buchmüller, Sandra (2010). G - Gender Inspired Technology. In Waltraud Ernst (Hrsg.), Geschlecht und Innovation. Gender-Mainstreaming im Techno-

Wissenschaftsbetrieb. Internationale Frauen- und Genderforschung in Niedersachsen. Teilband 4.

Berlin: LIT Verlag.

Joost, Gesche (2008). Gender-Aspekte in der Designausbildung. In Ingrid Hasper (Hrsg.). Key competence: Gender. HAWK-Ringvorlesung 2007/2008. Abgerufen am 28. Februar 2014 von http://www.hawk-hhg.de/hochschule/media/Genderaspekte_in_der_Designausbildung_v0.2.pdf

Klein-Franke, Silke (2006). Gender Aspekte in Forschungsvorhaben. Wozu? Forschung hat doch kein Geschlecht!. In Dorothea Erharter (Hrsg.), Gender Mainstreaming in Bildungseinrichtungen (S. 7391). Graz: Verein Forum Neue Medien.

Knoll, Bente (2014). Viele Facetten. Geschlechter- und diversityfreundliche Mediengestaltung in technischen Bereichen. In diesem Band.

Mohr, Nikolaus, Sauthoff-Bloch, Ann-Kathrin, Alt, Markus \& Derksen, Jens (2011). Mobile Web Watch 2011. Deutschland, Österreich, Schweiz. Die Chancen der mobilen Evolution. Abgerufen am 03. Januar 2013 von

http://www.accenture.com/SiteCollectionDocuments/Local_Germany/PDF/Accenture-Studie-MobileWeb-Watch-2011.pdf

Rat für Forschung und Technologieentwicklung (2005). FAS.research (Hrsg.) Excellente Netzwerke Abgerufen am 28. Februar 2014 von http://www.fas.at/download-document?gid=253

Scambor, Elli, Zimmer, Fränk (2012). Die intersektionelle Stadt. Bielefeld: transcript.

Silverstein, Michael, Sayre, Kate \& Butmann, John (2009). Women want More. New York: Harper Business.

Wolffram, Andrea (2006). Studentische Technikhaltungen als gender-sensitiver Indikator für Ressourcen und Belastungen in der Studieneingangsphase. In Dorothea Erharter (Hrsg), Gender Mainstreaming in Bildungseinrichtungen (S. 19-31). Graz: Verein Forum Neue Medien. 
